\title{
A Review on Machine Learning and Artificial Intelligence in Management of Storage
}

\author{
Swapnali Rajaram Pawar \\ M.Tech Student, Department of Computer Engineering, Bharati Vidyapeeth College of Engineering, Pune, India ${ }^{1}$
}

\begin{abstract}
Associations keep running at the speed of their information. While Artificial Intelligence (AI) and Machine Learning (ML) have a proceeding with history of taking care of conventional issues in design acknowledgment, AI and ML methods are quickly finding their place in business examination, where the examples being resolved may be more subtle. The effectiveness of these learning frameworks can characterize an association's upper hand. AI is being utilized or trialed for a scope of social insurance and research purposes, including recognition of sickness, administration of constant conditions, conveyance of wellbeing administrations, and medication revelation. AI can possibly help address vital wellbeing challenges, yet may be restricted by the nature of accessible wellbeing information, and by the powerlessness of AI to show some human attributes.
\end{abstract}

Keywords: Machine learning, Management of storage, Artificial Intelligence, Storage management in Machine Learning.

\section{I.INTRODUCTION}

In the course of recent decades Machine Learning has turned out to be one of the backbones of data innovation and with that, a fairly focal, yet generally concealed, some portion of our life. With the consistently expanding measures of information getting to be accessible there is valid justification to trust that shrewd information examination will turn out to be much more inescapable as a fundamental element for innovative advancement [1]. Machine Learning has for quite some time been executed over customary process structures, where throughput and latencies are dictated by coupling register and capacity through the same systems administration and capacity interconnects that serve different business applications. The expanding volume and speed of arriving information are focusing on these structures, regardless of whether for continuous handling of Internet-of-Things (IoT) telemetry, design acknowledgment in pictures or sound, or mining information from the distribution center to increase new bits of knowledge around an association's clients or business [2]. There is no generally concurred meaning of AI. The term extensively alludes to figuring innovations that take after procedures related with human insight, for example, thinking, learning and adjustment, tactile comprehension, and connection. Right now, most utilizations of AI are restricted, in that they are just ready to do particular undertakings or take care of pre-characterized issues. AI works in a scope of courses, drawing on standards and apparatuses, including from maths, rationale, and science. An essential element of contemporary AI advances is that they are progressively ready to understand changed and unstructured sorts of information, for example, common dialect content and pictures. Machine-learning has been the best sort of AI lately, and is the fundamental methodology of a large number of the applications right now in use. 3 Rather than following premodified directions, machine learning enables frameworks to find designs and infer its own particular standards when it is given information and new encounters [3].

\section{II.RELATED WORK}

\section{A.WHAT IS MACHINE LEARNING?}

Expressed basically, Machine Learning is the utilization of extraordinary calculations that are capable at recognizing designs in datasets. The more information there is for these calculations to investigate, the better the outcomes have a tendency to be. Information from which examples ought to be separated are gone through these calculations in a procedure called preparing. When designs develop, the outcomes can be utilized to distinguish comparative examples in new datasets. Voice acknowledgment calculations, for instance, can be prepared on enormous sound datasets to decide the correct examples to perceive helpful words. While varieties exist, there are two essential ways to deal with the preparation procedure. Using machine learning with proper storage management we can supervise machine to build software automatically only by giving it customer requirement. All coding should be done automatically by machine by learning Java , Python , C, C++ , R languages syntax from proper storage.eg-If user enters requirement -“create 
website ",then machine will ask user for language to use, website name, hyperlinks and other necessary details to build website and will build it.So that we can build small and simple application without need of developer.

To begin with, there is administered realizing, where human mediation is required to coordinate the coveted result of learning. Envision the demonstration of preparing a PC to perceive the photo of a feline. The calculations can be bolstered a huge number of varieties of feline pictures, alongside a few pictures that aren't felines, keeping in mind the end goal to teach it to perceive the catlike creature when given a photo of it later on. Managed learning depends on human (or machine) named datasets to tell the framework when it's a feline picture, and when it isn't, with the goal that it can learn.

Second, is unsupervised learning. As its name proposes, unsupervised learning investigates unlabeled datasets and distinguishes designs that may not be evident to people. This is a territory that is to a great degree dynamic in the business world today, as unsupervised learning can discover designs in client exchange information, wellbeing information, budgetary exchanging and numerous others. In spite of the distinctions in how learning may function for a given dataset and wanted result, there are extremely regular qualities in how frameworks are worked to ingest information, gain from it, and store it to learn again later on. The accompanying figure pleasantly exhibits the complexities engaged with the procedure from a framework work process viewpoint.

Figure 1: Modern Learning Pipeline

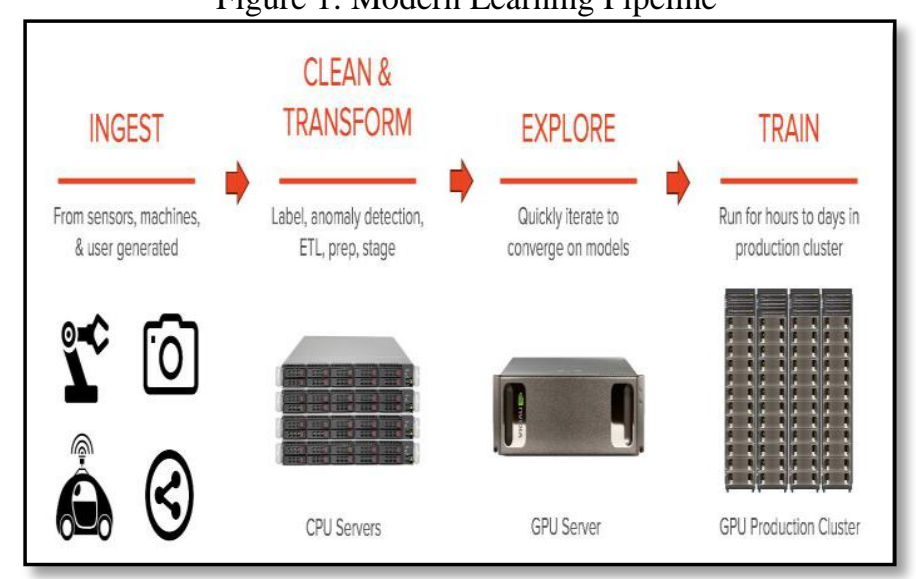

Source: Pure Storage

\section{B.MACHINE LEARNING WORKFLOWS-}

The initial step is ingesting the information that the preparation calculations will look at. Information is brought into the work process from outside. This could be from any number of sources. Possibly it's continuous information, from a variety of sensors, or it could be information created by business tasks and put away in the customary stockpiling exhibits in the venture datacenter. Regularly, it is information that was utilized in adapting beforehand, and tried again by the preparation calculations as they've advanced. In these cases, the information is moved from its source to industrious capacity to some degree closer to the learning condition.

Before being broke down by the learning calculations, the ingested information should be readied. This is the cleaning stage. Calculations might be kept running on it to recognize peculiarities, expel copy information, enhance the information with meta-information labels, or play out any number of different errands in view of the objectives of the investigation. This is information concentrated, however not in every case computationally burdening. The speed of the procedure relies upon the productivity of moving the information through the cleaning procedure.

When information is ingested and cleaned, the preparation calculations are relatively prepared to execute. Regularly various examinations will be kept running on the datasets to help set the phase for the real learning process. This is the investigation stage, where scientists work with the information to comprehend and help control the learning procedure to come.

At long last, there is the preparation arrange. There are various minor departure from what occurs amid this stage. The procedure can take anyplace from hours to weeks contingent upon the objectives of the learning. The essential thing to note is that it is computationally saddling, and the productivity (and length) of the procedure is a mix of the adequacy of the register motors, combined with the capacity to keep applicable information encouraged into them [2]. 
Vol. 7, Issue 9, September 2018

Here are eight particular stockpiling necessities of AI applications and why they request the information administration capacities provided by big business question stockpiling arrangements.

\section{SCALABILITY}

Artificial insight frameworks can process immense measures of information in a short time period-a fundamental quality since vast informational collections are required to convey precise calculations. This information volume drives critical capacity requests. Microsoft, for instance, required five long stretches of consistent discourse information to instruct PCs to talk. Tesla is instructing autos to drive with 1.3 billion miles of driving information. Dealing with these informational indexes requires a capacity framework that can scale unbounded.

\section{COST EFFICIENCY}

A helpful stockpiling framework must be both versatile and moderate, two traits that don't generally coincide in big business stockpiling. Verifiably, exceptionally adaptable frameworks have been more costly on a cost/limit premise. Expansive AI informational collections are not plausible in the event that they break the capacity spending plan.

\section{SOFTWARE-DEFINED STORAGE OPTIONS}

Tremendous informational collections will here and there require hyperscale server farms with purpose built server designs as of now set up. Different arrangements may profit by the effortlessness of pre-designed apparatuses.

\section{HYBRID ARCHITECTURE}

Distinctive information composes have shifting execution prerequisites, and the equipment must mirror that. Frameworks must incorporate the correct blend of capacity advances to meet the synchronous requirements for scale and execution, instead of a homogeneous methodology that will miss the mark.

\section{PARALLEL ARCHITECTURE}

For informational collections that develop unbounded, a parallel-get to design is basic. Something else, the framework will create gag focuses that cutoff development.

\section{DATA DURABILITY}

Support up a multi-petabyte preparing informational collection isn't constantly attainable; it would regularly be cost and time restrictive. However, you can't abandon it unprotected either. Rather, the capacity framework should act naturally securing.

\section{DATA LOCALITY}

While some AI information will residein the cloud, quite a bit of it will stay in the server farm for an assortment of reasons: execution, cost, and administrative consistence are three of them. To be focused, on-prem capacity must offer a similar expense and versatility benefits as its cloud-based partner.

\section{CLOUD INTEGRATION}

Notwithstanding where information lives, coordination with general society cloud will in any case be an essential prerequisite for two reasons. In the first place, a great part of the AI development is happening in the cloud. On-prem frameworks that are cloudintegrated will give the best adaptability to use cloud-local apparatuses. Second, we are probably going to see a liquid stream of information to/from the cloud as data is produced and broke down. An on-prem arrangement ought to rearrange that stream, not confine it [4].

\section{C.PROBLEMS OF MACHINE LEARNING AND STORAGE}

It requires an enormous measure of computational strength and information volume to process the present machine learning. It wasn't long prior that customary x86-based servers, upheld by conventional capacity exhibits and system 
connected capacity (NAS) gadgets, were sufficient to run learning calculations. These are, all things considered, similar servers and capacity arranges that endeavors trust to maintain their different business basic applications.

The ongoing transformation in machine learning, driven by profound learning, happened because of various joining advancements. Innovation touched base at a point where it could start to convey on the guarantee of computerized reasoning and machine learning tangibly. The business changed the specialty of the conceivable.

Measure turned out to be generally shoddy and copious. In-memory registering models quickly advanced to where, rather than getting information from outer capacity, spilling information is encouraged into indistinguishable area from the processors playing out the investigation. This permitted arrangements, for example, Apache Kafka, which merges various information sources to intermediary to the examination bunches, to advance.

In the meantime, the illustrations preparing unit (GPU) ended up about something beyond conveying quick activity to first individual gamers. For reasons unknown, the parallel preparing units in these GPUs are great at executing precisely the sorts of calculations required to fulfill the requests set forth by machine learning.

While quick processors are extraordinary, GPUs have truly been hard to program. As a pioneer in GPU advancement, NVIDIA attempted to decrease the weight of programming GPUs by improving a dialect considered CUDA and an extensive variety of devices to help the information researchers who build up the preparation calculations. The GPU programming biological system has bloomed, turning into the empowering agent for a heavenly body of new profound learning calculations [2].

$\mathrm{AI}$ and machine learning are impacting information stockpiling in a wide range of ways. Here are a portion of the best patterns and expectations:

\section{LOWER COSTS}

With ventures moving towards distributed storage and less committed stockpiling exhibits, dynamic stockpiling programming with incorporated profound learning calculations can assist association with gaining more stockpiling limit, at a 60 percent to 70 percent decrease in cost," .

\section{MORE SOFTWARE-DEFINED STORAGE}

Programming characterized capacity has been touted as a pattern for two or three years now. AI and machine learning are going about as accelerants. The numerous potential advantages are helping endeavors beat their hesitance to being told there is amazingly, one more new innovation to embrace.

\section{MORE INSTRUMENTATION:}

The beginning of registering saw a lot of instrumentation being added to frameworks. Truth be told, there are whole meetings and affiliations dedicated to the estimation and instrumentation of PCs. With Windows servers multiplying from the mid-1990s onwards, this side of the business has bit by bit faded. However, that gives off an impression of being changing as AI and machine learning open up new skylines. Therefore, Liebl predicts another pattern of significantly more prominent instrumentation in the years ahead.

\section{AUTOMATION:}

The coming of programming characterized capacity is a key impact in the ascent of machine learning and AI away situations. Including a heterogeneous programming control layer over the equipment enables the product to screen unmistakably assignments. This authorizes the capacity supervisor for more key obligations.

\section{ENHANCE SECURITY AND RELIABILITY}

Security and loss of are real worries for the cutting edge endeavor. Some stockpiling merchants are starting to outfit AI and machine figuring out how to avoid information misfortune, increment accessibility and speed turnaround amid downtime by means of brilliant information recuperation and precise reinforcement procedures, said Chaterji. He included this likewise guarantees better security. 


\section{HYBRID STORAGE CLOUDS}

The contention about open versus private mists has all the earmarks of being disputable even with AI, machine learning and programming characterized capacity. That is on account of utilitarian programming characterized designs ought to have the capacity to progress information consistently starting with one kind of cloud then onto the next. In the meantime, associations can deal with every one of their information as one pool, paying little respect to where it physically dwells. Accordingly, the perfectionists who look for all open or every private cloud are not prone to win. It is the half and half cloud that is well on the way to thrive.

\section{MORE FLASH}

Everybody predicts more blaze, so what's new? AI and machine learning will simply add yet more driving force to this relatively relentless wave that is clearing over all types of capacity.

\section{AUTOMATED CARS}

Maybe the greatest driver that will eventually give the utilization case to coordinating AI and machine learning into capacity will be drivers - auto drivers. The present top of the line autos (without independent highlights) have anyplace somewhere in the range of 64 and 200 GB of capacity - for the most part for maps and infotainment capacities. In tomorrow's independent vehicles, we may see in excess of $1 \mathrm{~TB}$ of capacity, and this won't simply be for the drive work.

\section{PARALLEL FILE SYSTEMS}

With a specific end goal to help AI and machine learning abilities, stockpiling frameworks should convey execution at scale. This implies they should have the capacity to function admirably at anticipated scale with advancements like parallel record frameworks and glimmer. So said Laura Shepard, senior executive, item advertising, DataDirect Networks.

\section{NEURAL STORAGE}

Liebl additionally estimates the ascent of "neural-class" stockpiling. This is the place the capacity can perceive and react to issues and openings without human intercession. At the point when that innovation grabs hold, expect a stage change in profitability.

Straight to the point Berry, an investigator at IT Brand Pulse, said touching base at neural capacity won't occur without any forethought. He spread out three stage coming full circle in the acknowledgment of neural stockpiling systems. They will show bit by bit and one will prompt the other. Stage 1 is, as specified by Liebl, where capacity is instrumented with telemetry to gather information from non-conventional sources. For instance, client level access designs, information streams, organizing streams, and information about equipment and programming disappointments. This stage shows in the generally beginning periods of programming characterized capacity.

Stage 2 is the thing that Berry alludes to as self-driving. When capacity is all product characterized, which calculations can wind up incorporated and sufficiently extensive to take care of complex stockpiling administration issues graciousness of the abundance of new information they can get to. This is an essential advance making progress toward building the observing, tuning, mending administration chains required for self-driving [5].

\section{III.CONCLUSION}

Machine learning is unpredictable, tedious and information substantial. Modelers and specialists actualizing the frameworks that convey on the guarantee of machine learning have a tendency to appropriately center around the intricate errand of coordinating CPUs and GPUs to help the calculations that will make their undertakings effective. Capacity and conveyance of information can significantly impact the productivity of a machine learning condition. Advancement in this world proceeds at a fast pace, and it's important that you converse with the pioneers who both see the present machine learning condition and are building tomorrow's. 


\section{IJARCCE}

\section{REFERENCES}

[1] https://www.kth.se/social/upload/539743e8f276543de71694a0/thebook.pdf

[2] Moor insights and strategy, "STORAGE-OPTIMIZED MACHINE LEARNING

[3] IMPACT OF STORAGE ON MACHINE LEARNING “Storage-Optimized Machine Learning February 2018 Copyright @2018 Moor Insights \& Strategy

[4] http://nuffieldbioethics.org/wp-content/uploads/Artificial-Intelligence-AI-in-healthcare-and-research.pdf

[5] Cloudian, Inc. 177 Bovet Road, Suite 450 San Mateo, CA 94402 Tel: 1.650.227.2380 Email: info@cloudian.com Web: cloudian.com

[6] http://www.enterprisestorageforum.com/storage-management/top-10-ai-and-machine-learning-data-storage-trends.html

[7] A. Khare and N. Jayakumar, "Perspective Analysis Recommendation System in Machine Learning," Int. J. Emerg. Trends Technol. Comput. Sci., vol. 6, no. 2, pp. 184-187, 2017.

[8] P. D. N. K. Akash U. Suryawanshi, "Review on Methods of Privacy-Preserving auditing for storing data security in cloud," Int. J. Adv. Res. Comput. Commun. Eng. (IJARCCE), ISSN, vol. 7, no. 4,pp.247-251,2018

[9] J. Namdeo and N. Jayakumar, "Predicting Students Performance Using Data Mining Technique with Rough Set Theory Concepts," Int. J. Adv. Res. Comput. Sci. Manag. Stud., vol. 2, no. 2, 2014

[10] K. S. RAVAL, R. S. SURYAWANSHI, J. NAVEENKUMAR, and D. M. THAKORE, "The Anatomy of a Small-Scale Document Search Engine Tool: Incorporating a new Ranking Algorithm," 2011.

[11] D. T. Jayakumar and R. Naveenkumar, “SDjoshi,“,” Int. J. Adv. Res. Comput. Sci. Softw. Eng. Int. J, vol. 2, no. 9, pp. 62-70, 2012.

[12] N.J.Prashant Desai, “AN EXTENSIBLE FRAMEWORK USING MOBILITYRPC FOR POSSIBLE DEPLOYMENT OF ACTIVE STORAGE ON TRADITIONAL STORAGE ARCHITECTURE,” IIOAB J., vol. 9, no. 3, pp. 25-30, 2018.

[13] D. N. K. J. Osho Tripathi Paras Gandhi, “GARDUINO- The Garden Arduino,” Int. J. Comput. Sci. Technol., vol. 8, no. 2, pp. $145-147,2017$.

[14] A. K. K. A. C. N. J. Divyansh Shrivastava Shubham Kesarwani, "Online Student Feedback Analysis System with Sentiment Analysis,” Int. J. Innov. Res. Sci. Eng. Technol., vol. 6, no. 5, pp. 8445-8451, 2017.

[15] P. D. N. kumar Yogesh Sawant, "Crisp Literature Review One andScalableFramework:Active Model to Create Synthetic Electrocardiogram Signals,” Int. J. Appl. or Innov. Eng. Manag., vol. 5, no. 11, pp. 73-80, 2016.

[16] N. J. R. Salunkhe A. D. Kadam and S. Joshi, ““'Luster A Scalable Architecture File System: A Research Implementation on Active Storage Array Framework with Luster file System," in ICEEOT, 2015.

[17] J. G. AyushKhare, N. Bhatt, and N. Kumar, "Raspberry Pi Home Automation System Using Mobile App to Control Devices," Int. J. Innov. Res. Sci. Eng. Technol., vol. 6, no. 5, pp. 7997-8003, 2017.

[18] P. R. Desai and N. K. Jayakumar, “A Survey on Mobile Agents,” Int. J. Res. Appl. Sci. Eng. Technol., vol. 5, no. XI, pp. $2915-2918,2017$.

[19] N. Jayakumar, "Active storage framework leveraging processing capabilities of embedded storage array," 2015.

[20] N. Jayakumar, M. S. Iyer, S. D. Joshi, and S. H. Patil, "A Mathematical Model in Support of Efficient offloading for Active Storage Architectures," in International Conference on Electronics, Electrical Engineering, Computer Science (EEECS) : Innovation and Convergence, 2016,vol.2,p.103.

\section{BIOGRAPHY}

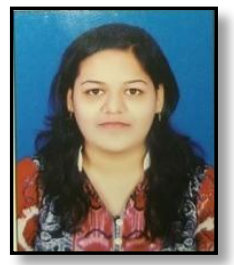

Swapnali Rajaram Pawar

Department of Computer Engineering, Bharati Vidyapeeth College of Engineering, Pune. 